\title{
Clip-line closure with the reopenable clip over line method for a large mucosal defect after gastric endoscopic submucosal dissection
}

Mucosal defects that occur following large gastric endoscopic submucosal dissection (ESD) are a cause of postoperative bleeding [1-3]. A standard method for large mucosal defect closure without dead space between the normal mucosa and mucosal defect has not yet been established. We invented clip-line closure with the reopenable clip over line method (ROLM) for complete closure of the mucosal defect left after gastric ESD.

In clip-line closure using ROLM, only reopenable clips (SureClip; 8 or $16 \mathrm{~mm}$; MC Medical, Tokyo, Japan) and a line $(0.23 \mathrm{~mm}$ nylon line) are used. First, the line is tied to one tooth of a reopenable clip, which is then inserted into the accessory channel without the endoscope being withdrawn ( Fig. 1; V Video 1). The clip is then positioned so that it grips the margin of the mucosal defect. Next, the end of the line exiting the accessory channel of the endoscope is passed through the hole in one tooth of a second reopenable clip, and this reopenable clip is inserted into the endoscope (the ROLM). The second clip is placed on the opposite side of the defect, with the muscular layer of the mucosal defect being grasped by the tooth of the reopenable clip through which the line has been passed. The teeth of the reopenable clips through which the line passes are therefore continuously fixed by the line, preventing the clips from being buried into the side of the muscle layer. Repeating ROLM gradually closes the mucosal defect from the anal to the oral side. Video 1 shows an in vitro model and a patient example of mucosal defect closure post-gastric ESD using clip-line closure with ROLM ( $>$ Fig. 2 ).

Clip-line closure using ROLM can completely close a large gastric mucosal defect without leaving dead space between the normal mucosa and the mucosal defect.

Endoscopy_UCTN_Code_TTT_1AQ_2AG
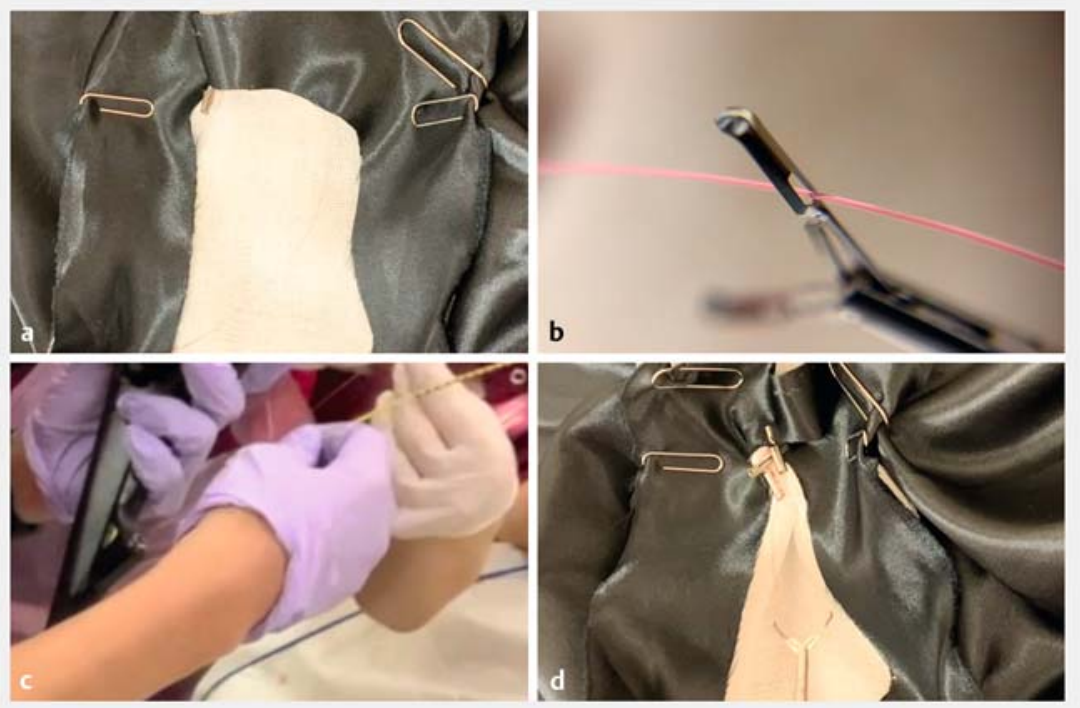

- Fig. 1 Images of an in vitro model demonstrating the steps for clip-line closure with the reopenable clip over line method (ROLM): a a clip with a line tied to it is placed on the margin of the mucosal defect, after being passed through the accessory channel of the endoscope; b the line from the accessory channel is passed through the hole in one of the teeth of a second reopenable clip; $\mathbf{c}$ with the line held by the assistant, the reopenable clip is inserted into the endoscope; $\mathbf{d}$ the reopenable clip is then placed on the contralateral margin of the mucosal defect and, with the use of ROLM, further reopenable clips are placed on alternating sides of the mucosal defect until the defect is gradually closed.

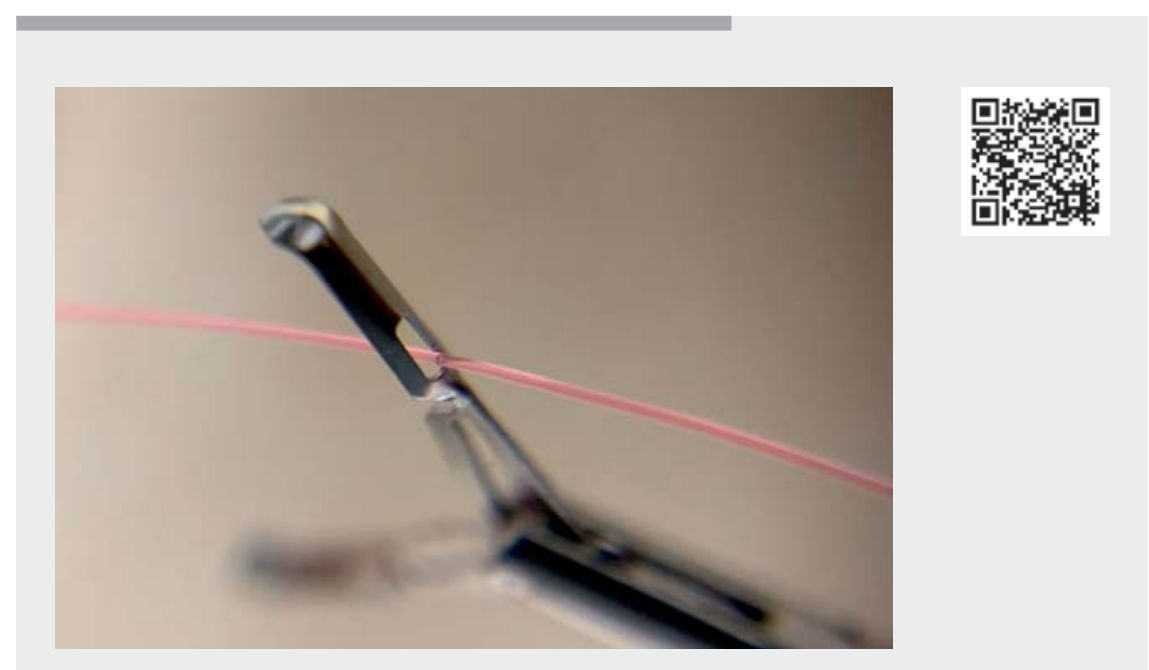

Video 1 Video showing an in vitro model of clip-line closure with the reopenable clip over line method (ROLM) and the performance of clip-line closure with ROLM for a large mucosal defect post-gastric endoscopic submucosal dissection. 


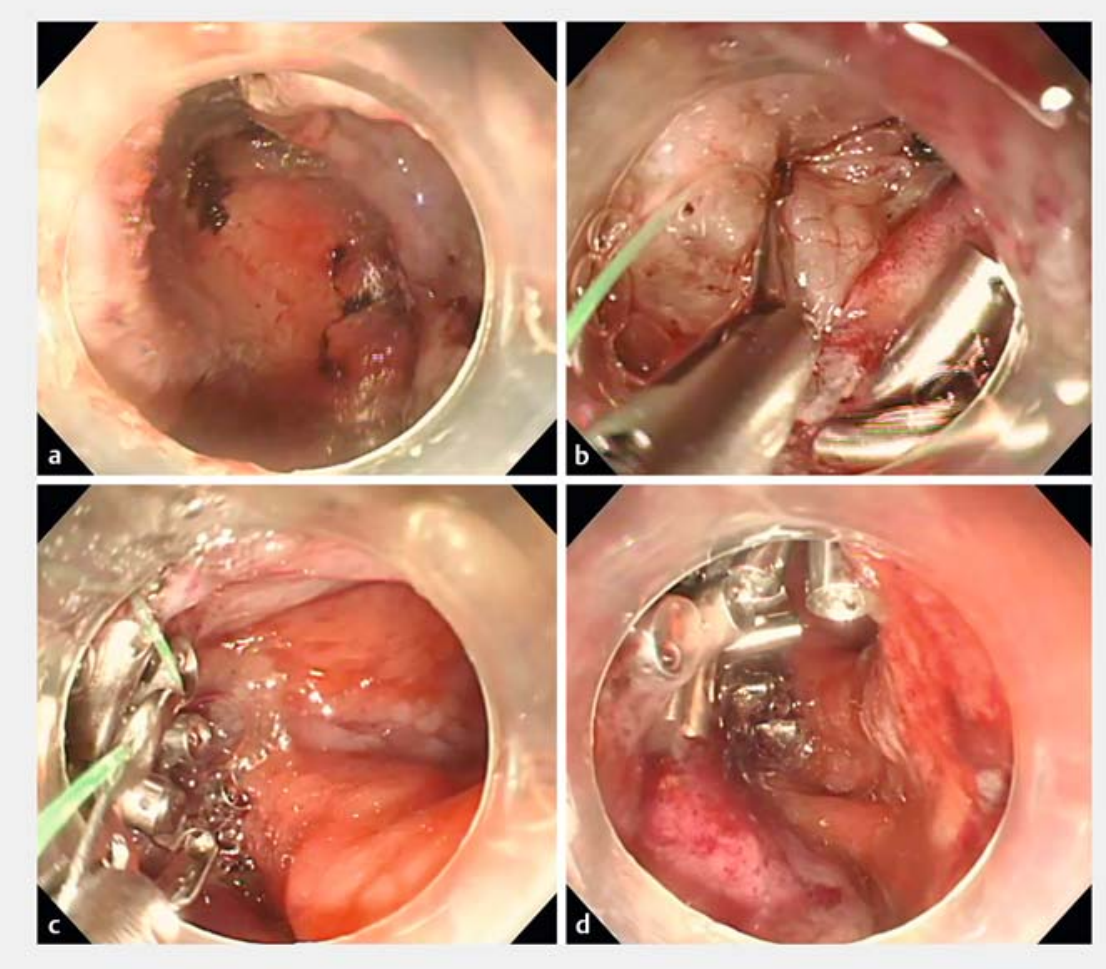

- Fig. 2 Endoscopic images of a mucosal defect post-gastric endoscopic submucosal dissection (ESD) being closed using clip-line closure with the reopenable clip over line method (ROLM) showing: a a mucosal defect of $85 \mathrm{~mm}$ after gastric ESD; $\mathbf{b}$ the normal mucosa and muscular layer at the margin of the mucosal defect being grasped by a reopenable clip with attached line; $\boldsymbol{c}$ the large mucosal defect being closed by repeating ROLM until the line is sutured in place without dead space between the normal mucosa and mucosal defect; $\mathbf{d}$ the mucosal defect after complete closure.

\section{Competing interests}

The authors declare that they have no conflict of interest.

\section{The authors}

Tatsuma Nomura ${ }^{1,2} \oplus$, Shinya Sugimoto², Taishi Temma ${ }^{2}$, Jun Oyamada ${ }^{2}$, Akira Kamei ${ }^{2}$

1 Department of Gastroenterology, Mie Prefectural Shima Hospital, Shima, Mie, Japan

2 Department of Gastroenterology, Ise Red Cross Hospital, Ise, Mie, Japan

\section{Tatsuma Nomura, MD}

Department of Gastroenterology, Mie Prefectural Shima Hospital, 1257 Ugata, m06076tn@icloud.com

\section{References}

[1] Takizawa K, Oda I, Gotoda T et al. Routine coagulation of visible vessels may prevent delayed bleeding after endoscopic submucosal dissection - an analysis of risk factors. Endoscopy 2008; 40: 179-183 Ago, Shima, Mie 517-0595, Japan
[2] Kataoka Y, Tsuji Y, Hirasawa K et al. Endoscopic tissue shielding to prevent bleeding after endoscopic submucosal dissection: a prospective multicenter randomized controlled trial. Endoscopy 2019; 51: 619-627

[3] Hatta W, Tsuji Y, Yoshio T et al. Prediction model of bleeding after endoscopic submucosal dissection for early gastric cancer: BEST-J score. Gut. doi:10.1136/gutjnl-2019319926

Bibliography

Endoscopy 2022; 54: E1-E2

DOI 10.1055/a-1346-8991

ISSN 0013-726X

published online 16.2 .2021

(c) 2021. Thieme. All rights reserved.

Georg Thieme Verlag KG, Rüdigerstraße 14, 70469 Stuttgart, Germany

\section{ENDOSCOPY E-VIDEOS \\ https://eref.thieme.de/e-videos}

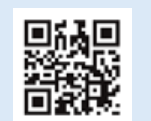

Endoscopy E-Videos is a free access online section, reporting on interesting cases and new techniques in gastroenterological endoscopy. All papers include a high quality video and all contributions are freely accessible online.

This section has its own submission website at

https://mc.manuscriptcentral.com/e-videos 